\title{
ANALYSIS OF EARLY STROKE-INDUCED CHANGES IN CIRCULATING LEUKOCYTE COUNTS USING TRANSCRIPTOMIC DECONVOLUTION
}

Growing evidence suggests that stroke alters the phenotype of the peripheral immune system; better characterization of this response could provide new insights into stroke pathophysiology. In this investigation, we employed a deconvolution approach to informatically infer the cellular composition of the circulating leukocyte pool at multiple timepoints following stroke onset based on whole blood mRNA expression. Microarray data generated from the peripheral blood of 23 cardiovascular disease controls and 23 ischemic stroke patients at 3,5 , and 24 hours post-symptom onset were obtained from a public repository. Transcriptomic deconvolution was used to estimate the relative counts of nine leukocyte populations based on the expression of cell-specific transcripts, and cell counts were compared between groups across timepoints. Inferred counts of lymphoid cell populations including B-cells, CD4+T-cells, CD8+T-cells, $\gamma \delta$ T-cells, and NK-cells were significantly lower in stroke samples relative to control samples. With respect to myeloid cell populations, inferred counts of neutrophils and monocytes were significantly higher in stroke samples compared to control samples, however inferred counts of eosinophils and dendritic cells were significantly lower. These collective differences were most dramatic in samples collected at 5 and 24 hours post-symptom onset. Findings were subsequently confirmed in a second dataset generated from an independent population of 24 controls and 39 ischemic stroke patients. Collectively, these results offer a comprehensive picture of the early stroke-induced changes to the complexion of the circulating leukocyte pool, and provide some of the first evidence that stroke triggers an acute decrease in eosinophil counts.

Keywords

Complete blood count $\cdot \mathrm{CBC} \cdot \mathrm{NLR} \cdot$ Neutrophil lymphocyte ratio $\cdot$ Immune suppression $\cdot$ WBC $\cdot$ White blood cell $\cdot$ WBC Differential $\cdot$ Eosinophil $\cdot$ Infection

\section{Introduction}

It is becoming increasingly evident that the peripheral immune system responds robustly to stroke, and that this response influences clinical outcome. For example, peripheral immune changes triggered by stroke are believed to contribute to the pathogenesis of adverse complications such as secondary tissue damage, hemorrhagic transformation, and post-stroke infection [1]. Thus, better characterization of the stroke-induced peripheral immune response could provide novel insights into stroke pathophysiology and open new avenues for immunotherapeutic intervention

Many studies which have investigated the peripheral immune response to stroke in humans have done so using the standard white blood cell differential collected as part of routine clinical evaluation; unfortunately, the clinical white blood cell differential provides quantification of a limited number of cell populations, often only total neutrophils, monocytes, and lymphocytes, and thus provides a relatively low-detail picture regarding peripheral immune status. Multicolor flow cytometry experiments have been used to examine more discrete subpopulations of leukocytes, however they have often only focused on small numbers of cell types in a single analysis. Thus, more detailed characterization of the stroke-induced changes to the cellular complexion of the peripheral immune system could reveal nuanced alterations which are pathologically relevant.

Several prior studies have performed genome-wide transcriptomic profiling of peripheral whole blood with the goal of identifying clinically-useful stroke biomarkers
Grant C. O'Connell*, Julia H.C. Chang

School of Nursing Case Western Reserve University, Cleveland, $\mathrm{OH}$
Received 09 August 2018 accepted 14 October 2018
[2-6]. Recent work by our group suggests that similar to other conditions [7], several of the gene expression changes observed between stroke patients and controls in these investigations were likely artifacts of underlying changes in leukocyte counts, and not true changes in transcription at the cellular level $[8,9]$. Transcriptomic deconvolution is a process which leverages such phenomena to informatically infer the cellular composition of complex biological samples based on aggregate gene expression through the analysis of cell-specific transcripts [10]. In this study, in an attempt to better characterize the stroke-induced peripheral immune response, we employed a deconvolution approach to infer the counts of nine major circulating leukocyte populations at multiple timepoints following stroke onset using publicly available human whole blood gene expression data. 


\section{Methods}

\section{Microarray data processing}

Raw microarray data generated from the peripheral whole blood of 23 controls, as well as 23 ischemic stroke patients at 3,5 , and 24 hours post-symptom onset, were downloaded as.CEL files from the National Center for Biotechnology Information (NCBI) Gene Expression Omnibus (GEO) via accession number GSE58294. Probe annotations were updated via the 'annotate' package for R ( $R$ project for Statistical Computing). Raw perfect match probe intensities were background corrected, quantile normalized, and summarized at the set level via robust multi-array averaging using the rma() function of the 'affy' package. Data were further summarized at the gene level via max intensity using the collapserows() function of the 'WGCNA' package.

\section{Deconvolution}

Estimated counts of B-cells, CD4+ T-cells, CD8+ T-cells, gamma delta $(\gamma \delta)$ T-cells, natural killer (NK) cells, monocytes, neutrophils, eosinophils, and dendritic cells were generated from normalized expression data using a list of 226 cell-specific genes (Figure 1) aggregated from a compendium of immune cell microarray data compiled by Newman et al. [11]. Weighted correlation network analysis was used to produce a relative count for each cell population based on the expression levels of its associated genes using the collapserows() function of the 'WGCNA' package according to the method described by Miller et al. [12]. Relative counts of each cell population were arbitrarily scaled from zero to one using unitybased normalization.

\section{Demographic information}

Clinical and demographic information associated with samples was aggregated from the descriptors reported in Stamnova et al. [13].

\section{Statistics}

All statistics were performed using $\mathrm{R}$ 3.3. Fisher's exact test was used for comparison of dichotomous variables. T-test or oneway ANOVA was used for comparisons of continuous variables where appropriate. The null hypothesis was rejected when $p<0.05$.

\section{Validation of results}

Findings were subsequently confirmed via deconvolution of a second publically available microarray dataset generated from an independent population of 24 controls and 39 ischemic stroke patients (GEO accession number GSE16561) [14].

\section{Results}

\section{Clinical and demographic characteristics}

Stroke samples originated from patients which were significantly older than control counterparts, but were well matched in terms of sex and ethnicity. In terms of risk factors for cardiovascular disease, groups were well matched with regards to rates of hypertension and diabetes, however control subjects displayed a significantly higher prevalence of dyslipidemia relative to stroke patients. All stroke patients had received thrombolytic intervention via recombinant tissue plasminogen activator (rtPA) following 3 hour blood collection (Table 1).

\section{Inferred leukocyte counts}

Inferred counts of lymphoid populations including B-cells, CD4+ T-cells, CD8+ T-cells, $\gamma \delta$ T-cell and NK-cells were all significantly reduced in stroke samples relative to controls (Figure 2A-E); this effect was most pronounced in samples collected at 5 and 24 hours following onset of symptoms. With regards to myeloid populations, inferred counts of neutrophils and monocytes were significantly higher in stroke samples in comparison to controls (Figure $2 \mathrm{~F}-\mathrm{G})$, however, inferred counts of dendritic cells and eosinophils were significantly lower (Figure $2 \mathrm{H}-1$ ). Once again, these differences were most pronounced at 3 and 5 hours postonset. An identical overall pattern of changes was observed in a second microarray dataset generated from an independent patient population (Supplemental Figure 1).

\section{Discussion}

Better characterization of the peripheral immune response to stroke could provide novel insights into stroke pathophysiology and identify new targets for immunotherapeutic intervention. In this study, we employed a transcriptomic deconvolution approach to infer the relative counts of nine major circulating leukocyte populations in blood samples collected at multiple timepoints over the first 24 hours following stroke onset. With respect to several cell types, our results confirmed the findings of prior cytometric studies, however, our analysis also revealed changes in other leukocyte populations that have yet to be widely reported on in human stroke.

Our observations suggest that the circulating counts of lymphoid cell populations are ubiquitously suppressed in response to stroke. This is consistent with prior cytometric investigations which have reported strokeinduced decreases in total lymphocyte counts [15-18], as well as reductions in counts of more discrete lymphoid subpopulations such as B-cells, CD4+ T-cells, CD8+ T-cells, and NK-cells $[15,16,18]$. To our knowledge, only three other human studies have reported on circulating $\gamma \delta$ T-cell counts in stroke; one reporting that $\gamma \delta$ T-cell counts are elevated [19], one reporting that they are unaffected [20], and one reporting that they are decreased [21]. Our results provide additional evidence suggesting that stroke triggers an acute decrease in circulating $\gamma \delta$ T-cell numbers similar to that which is observed in other lymphoid populations.

Our results are also consistent with those of several prior cytometry-based investigations reporting that counts of the two most abundant peripheral blood myeloid cell populations, neutrophils and monocytes, become robustly elevated in response to stroke $[15,17,22,23]$. However, our findings also suggest that two lesser-studied myeloid populations, dendritic cells and eosinophils, are significantly reduced. With respect to dendritic cells, our observations are consistent with those reported in the limited number of prior cytometric studies performed in humans [24]. With respect to eosinophils, this current study is one of the first case-control analyses to report evidence of an acute reduction in eosinophil counts in response to stroke; our observations align well with two recent associative studies reporting 

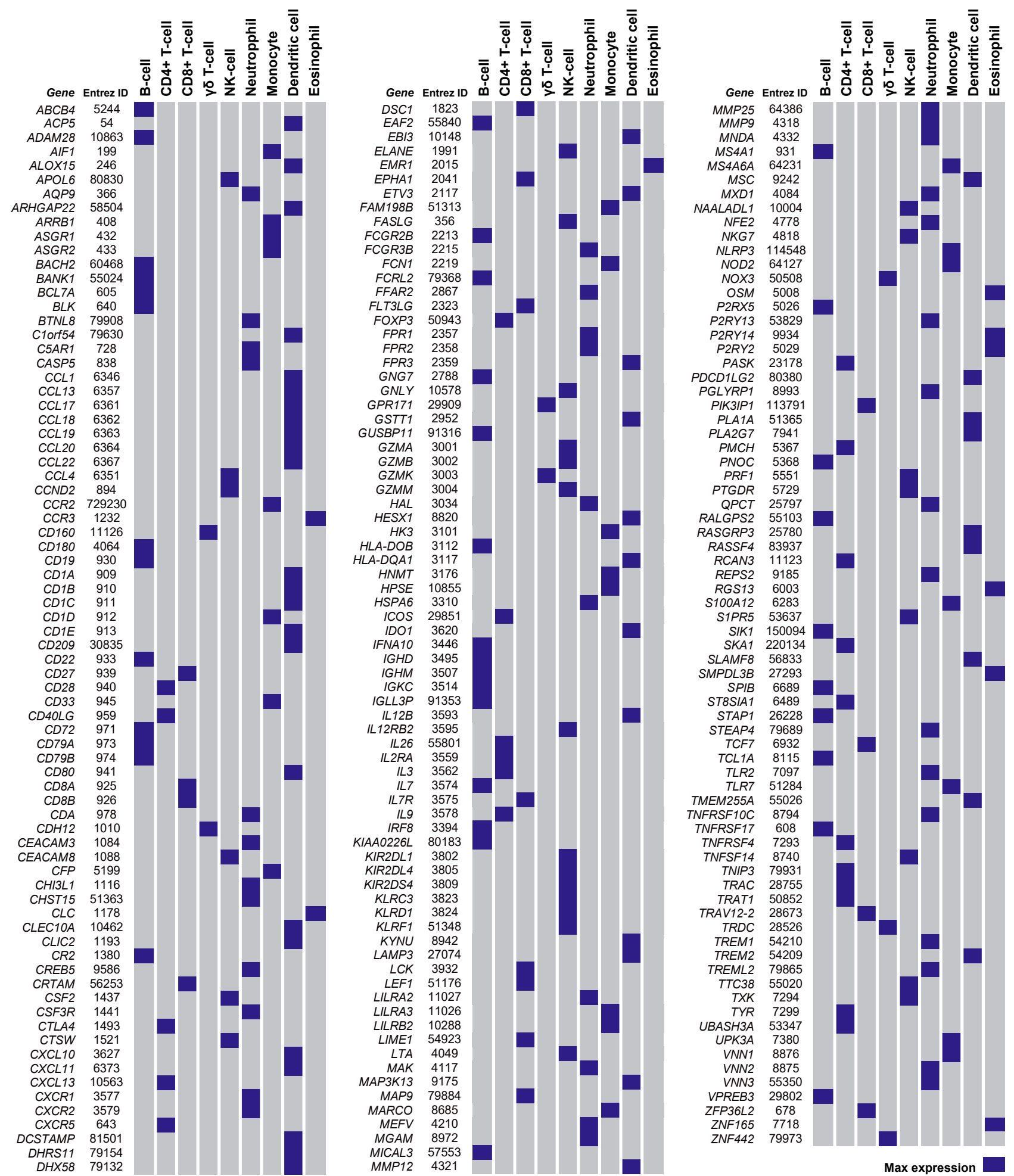

Figure 1. Cell-specific genes used for deconvolution. 226 cell-specific genes used for the deconvolution of whole blood microarray data along with their predominant leukocyte population of expression. 
Table 1. Clinical and demographic characteristics.

\begin{tabular}{|c|c|c|c|}
\hline & Cardiovascular Disease $(n=23)$ & Ischemic Stroke $(n=23)$ & p \\
\hline${ }^{\text {aAge }}$ mean $\pm S D$ & $57.9 \pm 3.3$ & $71.7 \pm 7.9$ & $<0.001^{*}$ \\
\hline${ }^{\mathrm{b} F e m a l e} n(\%)$ & $11(47.8)$ & $11(47.8)$ & 1.000 \\
\hline${ }^{b}$ Non-caucasian $n(\%)$ & $4(17.4)$ & $8(34.8)$ & 0.314 \\
\hline 'Dyslipidemia $n(\%)$ & $16(69.6)$ & $6(26.1)$ & $0.007^{*}$ \\
\hline${ }^{\text {bHypertension } n(\%)}$ & $16(69.6)$ & $16(69.6)$ & 1.000 \\
\hline 'Diabetes $n(\%)$ & $5(21.7)$ & $4(17.4)$ & 1.000 \\
\hline${ }^{a}$ Baseline NIHSS mean $\pm S D$ & $0.0 \pm 0.0$ & $15.4 \pm 7.4$ & $<0.001^{*}$ \\
\hline brtPA $n(\%)$ & $0(0.0)$ & $23(100.0)$ & $<0.001^{*}$ \\
\hline
\end{tabular}

A

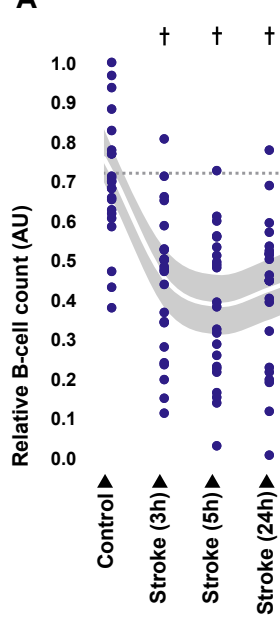

B

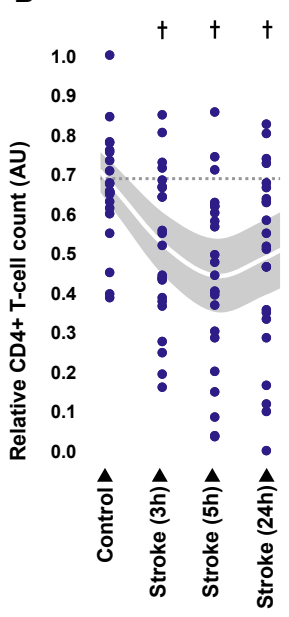

$\mathbf{F}$

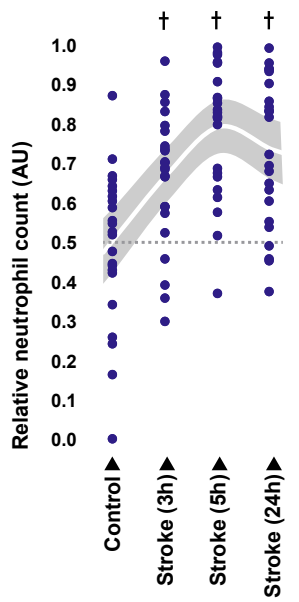

C

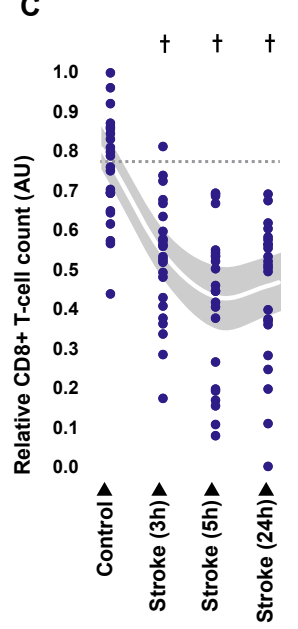

G

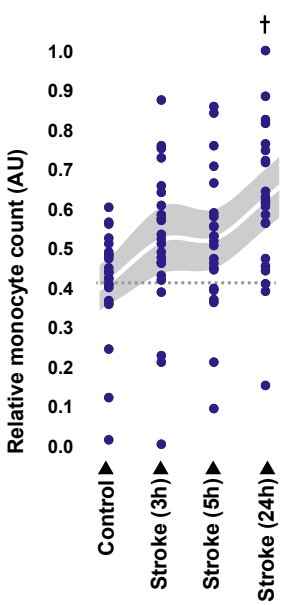

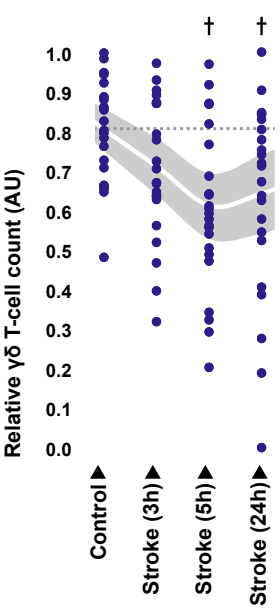

E

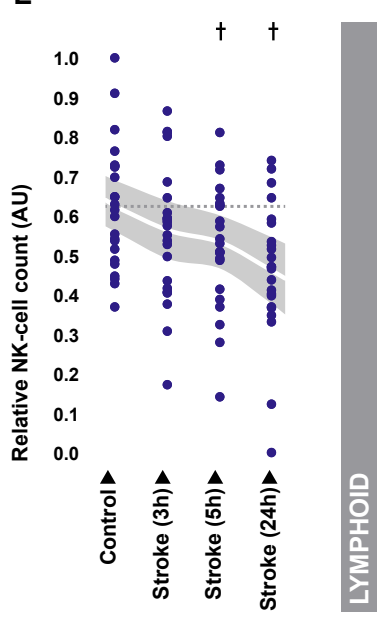

H

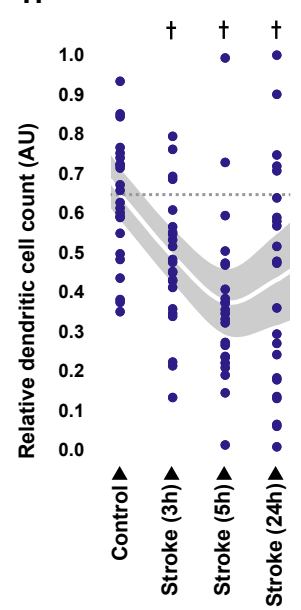

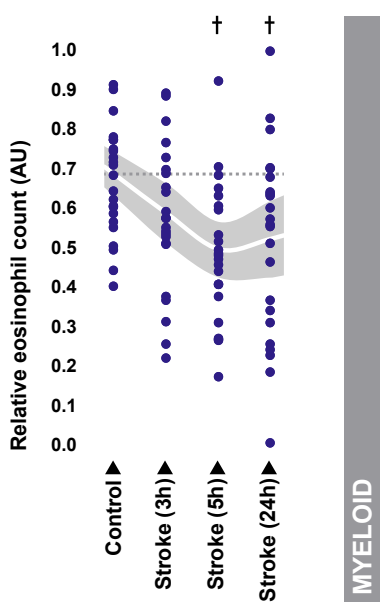

Figure 2. Inferred counts of circulating leukocyte populations. (A-l) Estimated relative counts of B-cells, CD4+ T-cells, CD8+ T-cells, $\gamma \delta$ T-cells, NK-cells, monocytes, neutrophils, eosinophils, and dendritic cells in blood sampled from controls and stroke patients at 3,5, and 24 hours post symptom onset. Counts were statistically compared between stroke and control samples across time points using one-way ANOVA with subsequent planned comparisons via Bonferoni-corrected two-sample two-tailed t-test. 
that post-stroke circulating eosinophil counts are negatively correlated with stroke severity and risk of mortality [25,26], suggesting that the pathophysiological relevance of a stroke-induced reduction in peripheral blood eosinophil counts warrants further investigation.

It should be stated that this study is not without limitations; most notable in this regard is the fact that we did not directly measure leukocyte counts, and instead informatically inferred them using gene expression data. While transcriptional deconvolution approaches have been shown to accurately enumerate cell counts in numerous benchmarking studies [10-12], the fact that they rely on reference expression signatures generated from isolated healthy cells has the potential to introduce confounds. The handling and manipulation of reference cells during isolation could potentially alter gene expression and introduce artifacts. Furthermore, because reference signatures are generated from the cells of healthy donors, disease-specific differential regulation of reference signature genes could reduce the accuracy of analyses. However, the aforementioned limitations are most likely to introduce confounds when trying to discriminate between highly similar cell populations or in disease states which introduce dramatic alterations in transcription; the cell types which were enumerated in our analysis are relatively distinct at the molecular level, and the magnitude of transcriptional changes observed in stroke at the level of whole blood are of a relatively small $[2,4,6]$. Furthermore, the fact that our findings align well with those of previous cytometry-based investigations suggests that our deconvolution analysis was well implemented. Nonetheless, our findings, in particular those regarding stroke-induced changes in eosinophil counts, should be confirmed in future work using direct cytometric analysis.

It is also important to note that because samples originated from patients who received thrombolytic treatment following the initial 3 hour blood collection, it is possible that some of the differences in inferred leukocyte counts observed between stroke patients and controls at the 5 and 24 hour timepoints were driven by effects of rtPA. However, we find this scenario unlikely due to the fact we observed a similar pattern of changes in a second dataset originating from blood samples which were collected prior to administration of thrombolytics.

Collectively, our results offer a comprehensive picture of the early stroke-induced changes to the complexion of the circulating leukocyte pool. Our findings confirm the results of prior cytometric investigations, and additionally, provide some of the first human evidence that stroke triggers an acute decrease in circulating eosinophil counts.

\section{Acknowledgements}

The authors would like to thank the members of FBP School of Nursing at Case Western Reserve University for providing general research support and critical review of the manuscript.

\section{Funding}

Work was funded via Case Western Reserve University FPB School of nursing start-up funds issued to GCO and a National Institute of Nursing Research T32 predoctoral fellowship awarded to JHCC (5T32NR15433-3).

\section{Conflict of interest}

GCO has a patent pending re: genomic patterns of expression for stroke diagnosis. GCO has received consulting fees from Valtari Bio incorporated. The remaining authors report no potential conflicts of interest.

\section{Compliance with ethical standards}

All procedures performed in studies involving human participants were in accordance with the ethical standards of the institutional and/or national research committee and with the 1964 Helsinki declaration and its later amendments or comparable ethical standards.

References

[1] Kamel $\mathrm{H}$, ladecola C. Brain-immune interactions and ischemic stroke: Clinical implications. JAMA Neurol. 2012;69:576-81.

[2] O'Connell GC, Petrone AB, Treadway MB, Tennant CS, Lucke-Wold N, Chantler PD, et al. Machine-learning approach identifies a pattern of gene expression in peripheral blood that can accurately detect ischaemic stroke. Npj Genomic Med. 2016;1:16038-16038.

[3] O'Connell GC, Treadway MB, Petrone AB, Tennant CS, Lucke-Wold $\mathrm{N}$, Chantler PD, et al. Peripheral blood AKAP7 expression as an early marker for lymphocyte-mediated post-stroke blood brain barrier disruption. Sci Rep. 2017;7:1172-1172.

[4] Stamova B, Xu H, Jickling G, Bushnell C, Tian Y, Ander BP, et al. Gene expression profiling of blood for the prediction of ischemic stroke. Stroke J Cereb Circ. 2010;41:2171-7.

[5] Jickling GC, Ander BP, Stamova B, Zhan X, Liu D, Rothstein L, et al. RNA in blood is altered prior to hemorrhagic transformation in ischemic stroke. Ann Neurol. 2013;74:232-40.
[6] Tang Y, Xu H, Du X, Lit L, Walker W, Lu A, et al. Gene expression in blood changes rapidly in neutrophils and monocytes after ischemic stroke in humans: a microarray study. J Cereb Blood Flow Metab Off J Int Soc Cereb Blood Flow Metab. 2006;26:1089-102.

[7] Whitney AR, Diehn M, Popper SJ, Alizadeh AA, Boldrick JC, Relman $D A$, et al. Individuality and variation in gene expression patterns in human blood. Proc Natl Acad Sci U A. 2003;100:1896-901.

[8] O'Connell GC, Treadway MB, Tennant CS, Lucke-Wold N, Chantler PD, Barr TL. Shifts in Leukocyte Counts Drive the Differential Expression of Transcriptional Stroke Biomarkers in Whole Blood. Transl Stroke Res [Internet]. 2018 [cited 2018 Jun 14]; Available from: http://link. springer.com/10.1007/s12975-018-0623-1

[9] O'Connell GC, Treadway MB, Petrone AB, Tennant CS, Lucke-Wold N, Chantler PD, et al. Leukocyte Dynamics Influence Reference Gene Stability in Whole Blood: Data-Driven qRT-PCR Normalization Is a Robust Alternative for Measurement of Transcriptional Biomarkers. 
Lab Med [Internet]. 2017;48. Available from: http://fdslive.oup.com/ www.oup.com/pdf/production_in_progress.pdf

[10] Abbas AR, Wolslegel K, Seshasayee D, Modrusan Z, Clark HF. Deconvolution of Blood Microarray Data Identifies Cellular Activation Patterns in Systemic Lupus Erythematosus. Tan P, editor. PLoS ONE. 2009;4:e6098.

[11] Newman AM, Liu CL, Green MR, Gentles AJ, Feng W, Xu Y, et al. Robust enumeration of cell subsets from tissue expression profiles. Nat Methods. 2015;12:453-7.

[12] Miller JA, Cai C, Langfelder P, Geschwind DH, Kurian SM, Salomon $\mathrm{DR}$, et al. Strategies for aggregating gene expression data: The collapseRows R function. BMC Bioinformatics. 2011;12:322.

[13] Stamova B, Jickling GC, Ander BP, Zhan X, Liu DZ, Turner R, et al. Gene expression in peripheral immune cells following cardioembolic stroke is sexually dimorphic. PLoS ONE. 2014;9:1-9.

[14] Barr TL, Conley Y, Ding J, Dillman a, Warach S, Singleton a, et al. Genomic biomarkers and cellular pathways of ischemic stroke by RNA gene expression profiling. Neurology. 2010;75:1009-14.

[15] Vogelgesang A, Grunwald U, Langner S, Jack R, Bröker BM, Kessler C, et al. Analysis of lymphocyte subsets in patients with stroke and their influence on infection after stroke. Stroke. 2008;39:237-41.

[16] Urra X, Cervera Á, Villamor N, Planas a. M, Chamorro Á. Harms and benefits of lymphocyte subpopulations in patients with acute stroke. Neuroscience. 2009;158:1174-83.

[17] Haeusler KG, Schmidt WUH, Föhring F, Meisel C, Helms T, Jungehulsing GJ, et al. Cellular Immunodepression Preceding Infectious Complications after Acute Ischemic Stroke in Humans. Cerebrovasc Dis. 2008;25:50-8.

[18] Liu Q, Jin W-N, Liu Y, Shi K, Sun H, Zhang F, et al. Brain Ischemia
Suppresses Immunity in the Periphery and Brain via Different Neurogenic Innervations. Immunity. 2017;46:474-87.

[19] He $Y$, Ma N, Xing C, Wang X, Xiao H, Zheng $M$, et al. Novel IL-6secreting $\gamma \delta \mathrm{T}$ cells increased in patients with atherosclerotic cerebral infarction. Mol Med Rep. 2015;11:1497-503.

[20] Peterfalvi A, Molnar T, Banati M, Pusch G, Miko E, Bogar L, et al. Impaired function of innate T lymphocytes and NK cells in the acute phase of ischemic stroke. Cerebrovasc Dis Basel Switz. 2009;28:4908.

[21] Adamski MG, Li Y, Wagner E, Yu H, Seales-Bailey C, Durkin H, et al. Pre-Existing Hypertension Dominates $\gamma \delta \mathrm{T}$ Cell Reduction in Human Ischemic Stroke. Lees JR, editor. PLoS ONE. 2014;9:e97755.

[22] Hug A, Dalpke A, Wieczorek N, Giese T, Lorenz A, Auffarth G, et al. Infarct Volume is a Major Determiner of Post-Stroke Immune Cell Function and Susceptibility to Infection. Stroke. 2009;40:3226-32.

[23] Urra X, Cervera Á, Obach V, Climent N, Planas AM, Chamorro A. Monocytes are major players in the prognosis and risk of infection after acute stroke. Stroke. 2009;40:1262-8.

[24] Yilmaz A, Fuchs T, Dietel B, Altendorf R, Cicha I, Stumpf C, et al. Transient decrease in circulating dendritic cell precursors after acute stroke: potential recruitment into the brain. Clin Sci. 2009;118:14757.

[25] Wang J, Ma L, Lin T, Li S-J, Chen L-L, Wang D-Z. The significance of eosinophils in predicting the severity of acute ischemic stroke. Oncotarget [Internet]. 2017 [cited 2018 Jun 14];8. Available from: http://www.oncotarget.com/fulltext/22199

[26] Guo L-B, Liu S, Zhang F, Mao G-S, Sun L-Z, Liu Y. The role of eosinophils in stroke: a pilot study. Eur Rev Med Pharmacol Sci. 2015;19:3643-8. 